\title{
Origins of Goldschmidt's atmophile elements and models of planetary accretion - V.M. Goldschmidt Medal Lecture \\ BERNARD MARTY
}

CRPG Université de Lorraine

Presenting Author: bernard.marty@univ-lorraine.fr

Hydrogen, carbon, nitrogen and oxygen (HCNO) are essential ingredients to build habitable worlds like Earth, but their origin and evolution on the terrestrial planets remains highly debated. Due to their inert and highly volatile nature, noble gases, (He, $\mathrm{Ne}, \mathrm{Ar}, \mathrm{Kr}$ and $\mathrm{Xe}$ ) constitute a powerful set of physical tracers that are often studied conjointly with HCNO. Some $4.567 \mathrm{Ga}$, the solar system formed from the collapse of a molecular cloud of gas (99 wt.\%) and dust (1wt.\%) in which HCNO and light noble gases were by far the most abundant elements [1]. In contrast, these elements are extremely depleted in terrestrial planets, making at most $0.1 \%$ of Earth's mass [2]. Furthermore, the relative isotope compositions of $\mathrm{HCNO}$ and noble gases in planetary bodies are markedly different from the starting composition of the protosolar nebula (PSN) from which planetary building blocks formed $[3,4]$. Therefore, direct trapping of nebular gas into solid materials cannot have been the primary source of planetary volatiles which must have originated from intermediate cosmochemical reservoirs that had been isotopically processed and transported to the inner planet forming region. Two different lines of models are advocated for the building of terrestrial planets. The classical view proposes oligarchic construction of intermediate planetary bodies, planetesimals and Mars-sized embryos over prolongated periods of time of several tens of Myr [5]. The pebble accretion model advocates direct building of Venus, Earth and Mars from mmsized dust over a few Myr [6]. The comparison of the elemental and isotopic compositions of atmophile elements in the terrestrial atmosphere and in the mantle with those of primitive reservoirs (solar, chondritic, cometary), permit to set stringent constraints on models of solar system dynamics and planetary accretion.

[1] Lodders ( 2003) Ap J 591, 1220

[2] Marty (2012) EPSL 313-314, 56

[3] Alexander et al. ((2012) Science 337, 721

[4] Piani et al. (2020) Science 369, 1110

[5] Raymond et al. (2009) Icarus 203, 644

[6] Johansen et al. (2021) Sci. Adv. 7, eabc0444 